\title{
The Evolution of Computational Hemodynamics as a Clinical Tool in Decision Making, Patient Specific Treatment and Clinical Management
}

The study of cardiovascular flows is of great interest because of the role of hemodynamics in cardiovascular disease, the single largest cause of death worldwide and responsible for more than half of mortality in the developed countries. The precise knowledge and understanding of local blood flow features in patientspecific simulations has emerged as a promising factor in clinical decision making, clearly requiring the development of tools for the accurate analysis and interpretation of blood flow dynamics. Methods of characterization of blood flow dynamics allow the investigation of challenging and clinically relevant problems and harness the power of biofluid mechanics analysis to solve problems at the clinical forefront.

The rationale for this Special Issue is to reinforce the clinical utility of computational hemodynamics, contributing to its translation into clinical practice, and increasing the awareness of the potential of hemodynamic modelling and quantitative data provided in silico. The increasing availability and efficiency of computational tools for patient-specific simulations will accelerate the rate of building large datasets, providing quantitative data useful for proofof-concept studies and for providing mechanistic explanations to clinical observations. Hopefully, this will enable clinicians to gain insights, develop intuitions, and provide constructive feedback and guidance for the development of more representative models. Moreover, the increasing sophistication of therapeutic solutions for cardiovascular pathologies require the development of tools for quantitative patient-specific simulations to aid therapeutic planning through the assessment of pre-operative scenarios and the prediction of therapeutic intervention outcomes.

In our opinion, this Special Issue highlights the enormous potential of computational hemodynamics toward providing: (1) objective, quantitative, and mechanism-based parameters useful for the study of the origin, development and progression of cardiovascular diseases, (2) patient-specific prediction of the outcome of therapeutic interventions, and (3) the design and optimization of implants and devices, and their effects on physiologic function. Specifically, methods for the reduction of the inherent complexity of blood flow fields and their relation with biological mechanisms are reviewed by Liu et al., Mohamied et al., Pedrizzetti \& Domenichini, and Shadden \& Arzani. This approach is also found in the studies proposed by Schrauwen et al. and Gallo et al. The development and progression of coronary pathologies are considered by the studies of Malvè et al., Timmins et al., and Wang et al. The potential of CFD in the improvement of diagnostic capabilities for aneurysms is discussed in Chung \& Cebral and specifically the case of abdominal aortic aneurysms in Xenos et al. The reproducibility of CFD studies in both treated and untreated cerebral aneurysms is assessed in Cito et al., presenting the results of the Virtual Intracranial Stenting Challenge 2011. Contributions to the assessment and the prediction of treatment outcomes for aortic pathologies are given by Goubergrits et al. and Cheng et al. The multi-

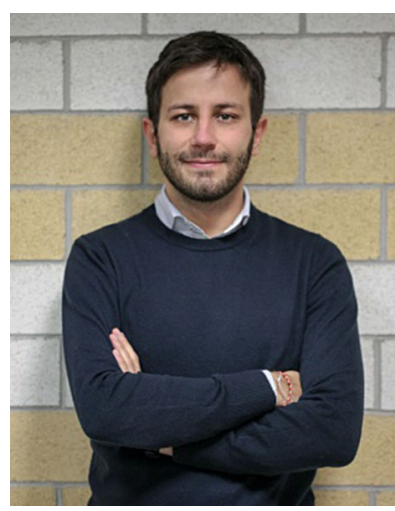

Diego Gallo.

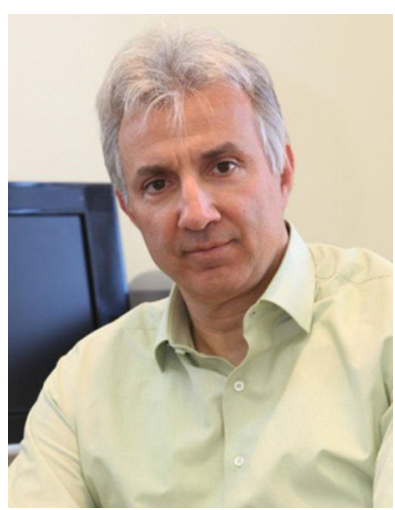

Andreas Anayiotos.

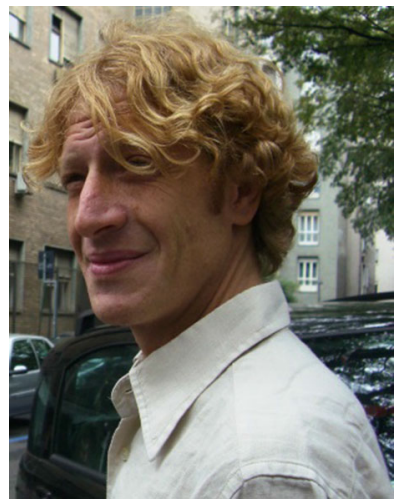

Umberto Morbiducci. 
scale nature of the phenomena involved in the pathophysiology of the cardiovascular system is featured through the review by Willemet \& Alastruey, and the contributions by Guala et al. and Sarrami-Foroushani et al., who make use of 1-D modelling. On the other hand, computational studies from the single cellular level to mesoscopic flow are reviewed by Omori and colleagues.

The 18 articles in this Issue comprise 7 review articles and 11 original research articles and represent in our opinion a comprehensive and timely highlight of the current research carried out by various research groups from all over the world. We hope that you will find this Special Issue informative, stimulating and thought-provoking. Finally, we would like to thank all the Reviewers that contributed to the overall quality of this Issue and the Annals of Biomedical Engineering for providing the platform for the communication of the advances on this exciting and promising field.
Diego Gallo

Department of Mechanical and Aerospace Engineering, Politecnico di Torino, Turin, Italy

Electronic mail: diego.gallo@polito.it

Andreas Anayiotos

Department of Mechanical Engineering and Materials Science and Engineering, Cyprus University of Technology, Limassol, Cyprus Electronic mail: andreas.anayiotos@cut.ac.cy

UMBERTO MORBIDUCCI

Department of Mechanical and Aerospace Engineering, Politecnico di Torino, Turin, Italy Electronic mail: umberto.morbiducci@polito.it 